\title{
Electrophysiological effects of flecainide and propafenone on atrial fibrillation cycle and relation with arrhythmia termination
}

\author{
M Biffi, G Boriani, G Bronzetti, A Capucci, A Branzi, B Magnani
}

\begin{abstract}
Objectives-(1) To investigate the electrophysiological effects of flecainide and propafenone during atrial fibrillation, and their relation to arrhythmia termination; (2) to investigate the effects of isoprenaline on atrial fibrillation in basal conditions and during treatment with class $1 \mathrm{C}$ drugs to evaluate the role of adrenergic stimulation on proarrhythmic events occurring during this treatment.

Design-Prospective, single centre study. Setting-University hospital.
\end{abstract}

Methods-10 patients with lone paroxysmal atrial fibrillation underwent an electrophysiological study. The dynamic behaviour of MFF (the mean of 100 consecutive atrial fibrillation intervals) was evaluated at two atrial sites after induction of atrial fibrillation either at baseline or after class $1 \mathrm{C}$ drug administration (flecainide or propafenone $2 \mathrm{mg}$ / $\mathrm{kg}$ ). The effects of isoprenaline on MFF and $R R$ interval were also investigated both under basal conditions and during class $1 \mathrm{C}$ drug treatment.

Results-After induction of atrial fibrillation, mean (SD) MFF shortened with time, and was further shortened by isoprenaline infusion (177 (22) $v 162$ (16) $v$ 144 (11) ms, p < 0.05). The administration of class $1 \mathrm{C}$ drugs reversed this trend and significantly increased the MFF to an average of 295 (49) ms, leading to conversion to sinus rhythm within 10 minutes in all patients. Atrial fibrillation was then reinduced on class $1 \mathrm{C}$ drugs: isoprenaline shortened the MFF and RR interval with a trend to AV synchronisation (223 (43) $v 269$ (49) ms for the MFF, 347 (55) $v 509$ (92) ms for the RR, $p<0.05)$; $1: 1$ sustained AV conduction occurred in two patients, at 187 and 222 beats/min respectively. One of these patients underwent electrical cardioversion because of haemodynamic collapse.

Conclusions-Class 1C drugs are effective at restoring sinus rhythm by increasing the MFF to a much greater extent than observed in self terminating atrial fibrillation episodes, and reversing the spontaneous atrial fibrillation behaviour (progressive shortening of MFF and self perpetuation of atrial fibrillation). MFF prolongation with 1:1 conduction at fast ventricular rates may lead to synchronisation during adrenergic stimulation, with a very short ventricular cycle; hence it is advisable to keep the patients at rest after acute class $1 \mathrm{C}$ drug loading or to consider pharmacological modulation of AV conduction for patients who are prone to a fast ventricular response.

(Heart 1999;82:176-182)

Keywords: atrial fibrillation; electrophysiology; flecainide; propafenone; isoprenaline

Although basic and experimental research has provided insight into the mechanisms of atrial fibrillation and the effects of many antiarrhythmic drugs, ${ }^{1-5}$ the determinants of pharmacological action during atrial arrhythmias are only partly understood. Class 1C antiarrhythmic drugs are useful treatment for recent onset atrial fibrillation in all the subsets of patients, ranging from those with lone atrial fibrillation to those with structural heart disease. ${ }^{6-8}$ Nonetheless their actions during atrial fibrillation require clarification, as they may not be identical to those predicted by the experimental environment. From the concept of wavelength ${ }^{2}$ one would not expect them to have such a striking effect in conversion to sinus rhythm because of their marked depression of conduction velocity. We showed previously that atrial dynamic changes during fibrillation describe the likelihood of self termination or persistence of the arrhythmia in patients with lone atrial fibrillation. ${ }^{9}$ We therefore sought to determine whether the same approach could identify the particular electrophysiological variables that are affected by class 1C agents in restoring sinus rhythm. We also examined the electrophysiological effect of adrenergic stimulation by isoprenaline during atrial fibrillation in patients given class 1C drugs, as the most important untoward effects (namely synchronisation with $1: 1$ atrioventricular conduction) have been described in patients experiencing some degree of adrenergic activation. ${ }^{1011}$

\section{Methods}

STUDY POPULATION

The study population comprised 10 patients (nine male and one female) ranging in age from 29 to 46 years (mean (SD) 36 (5) years) referred to our institution between July 1993 and November 1994 for frequent self terminating episodes of lone paroxysmal atrial fibrillation - that is, without any demonstrable organic heart disease. Atrial fibrillation had been documented previously on multiple Holter recordings and on hospital admissions, and 
these patients were referred because of weekly recurrent episodes of atrial fibrillation. Careful clinical history taking and repeated Holter recordings of the clinical arrhythmia did not identify any peculiar pattern leading to the onset of atrial fibrillation in any of the patients. Each patient underwent clinical evaluation, bidimensional echocardiography, a stress test, stress thallium 201 tomography, and determination of plasma thyroid hormone concentrations. All these tests needed to be normal for enrolment in the study.

Every drug that could interfere with atrial electrophysiological properties was discontinued at least five half times before the start of the study. No patient was evaluated under or after amiodarone treatment.

\section{ELECTROPHYSIOLOGICAL STUDIES}

Electrophysiological studies were carried out in a standardised way, as described previously in detail. ${ }^{9}$ Endocavitary atrial signals at the high right atrium (HRA), coronary sinus (CS), and right ventricle, and ECG leads $\mathrm{I}, \mathrm{aVF}, \mathrm{V} 2$, and V5 were simultaneously recorded on a seven channel recorder at a standard paper speed of $100 \mathrm{~mm} / \mathrm{s}$. Programmed stimulation was performed using a square wave at 2.5 diastolic threshold and $2 \mathrm{~ms}$ duration. The stimulation protocol included the single extrastimulus method at basic cycle length and at incremental cycle lengths of 600,500 , and $400 \mathrm{~ms}$. Atrial vulnerability was assessed by programmed electrical stimulation with one extrastimulus during sinus rhythm and atrial pacing at the three above mentioned cycle lengths.

The extrastimulus was delivered after eight paced beats late in diastole, and the coupling interval was shortened by steps of $5 \mathrm{~ms}$ and finally of $1 \mathrm{~ms}$ until the effective atrial refractory period was reached or atrial fibrillation was induced.

DATA ACQUISITION AND SIGNAL PROCESSING Two body surface ECG leads were recorded simultaneously with two atrial electrograms (HRA and CS) on a seven channel FM tape recorder and later digitised $(1 \mathrm{kHz}$ sampling frequency) for interval measurement. A computer program was developed to measure beat to beat atrial intervals (FF) from each recording site. The computer algorithm was divided in two main steps: atrial wave detection and local activation time measurement. For atrial wave detection an adaptive threshold on the filtered intra-atrial signal was used. ${ }^{12}$ To avoid baseline drift the algorithm first performs an incremental difference and then an energy collection is applied to enhance atrial waves. An atrial activation wave is detected when the filtered signal exceeds a threshold.

To follow morphological changes of the atrial signal, the threshold level and the other algorithm variables are beat to beat updated. For each detected atrial activation wave the local activation time was determined on the original signal, with $1 \mathrm{~ms}$ resolution, by specific algorithms. For bipolar recordings the algorithm searches for the greatest amplitude of the atrial wave deflection, whereas for unipolar electrograms local activation time was measured on the steepest intrinsic deflection. To prevent double counting, detection was discontinued for a suitable lag period after detecting a local activation.

The electrograms were displayed on a computer graphic screen together with the local activation times to check for errors or missing activation marks. The operator could correct the computer output by deleting or adding activation times. In few cases where the automatic procedure had too many faults, the atrial intervals were measured manually on the computer screen by positioning a cursor on the local atrial activation waves.

The RR interval sequence was also measured automatically by a similar procedure. ${ }^{2}$ Blood pressure was monitored through a femoral arterial sheath in all the patients.

\section{VARIABLES EVALUATED}

Basic electrophysiological variables, corrected sinus node recovery time (cSNRT), atrial and AV node refractoriness, and atrial vulnerability indices were calculated and have been reported elsewhere. ${ }^{9}$ In brief, basic electrophysiological variables were normal, whereas short atrial refractoriness, inhomogeneous atrial conduction times, and short latent vulnerability index during programmed atrial stimulation were observed in every patient. ${ }^{9}$

The diagnosis of atrial fibrillation was based on the surface ECG if there were no discrete $P$ waves in any surface lead and $F$ waves that were irregular in timing and morphology at a rate greater than 320 beats $/ \mathrm{min}$. These criteria were validated from the endocardial recordings by detecting irregular atrial activation, not separated by an isoelectric line (except for Wells type I atrial fibrillation) ${ }^{13}$ and with a standard deviation of the FF interval greater than $10 \mathrm{~ms}$. Moreover, RR intervals had to be irregular, and no periodic pattern of the FF intervals had to be present. ${ }^{14} 15$

To ensure a relatively stable situation, we evaluated only fibrillation episodes lasting longer than five minutes because these were not self terminating and allowed the study protocol to be completed (each patient had already had at least two atrial fibrillation episodes induced, which were self terminating within five minutes). At every recording site we measured 100 consecutive FF intervals (MFF) and the corresponding RR intervals, both at the onset and at the fifth minute of each long lasting atrial fibrillation episode. An isoprenaline infusion was then started at $5 \mu \mathrm{g} / \mathrm{min}$ and measurements were repeated at steady state.

After isoprenaline washout, flecainide or propafenone were given in random order and measurements were repeated at arrhythmia termination or after 10 minutes. Both drugs were given intravenously at a dose of $2 \mathrm{mg} / \mathrm{kg} /$ min over three minutes. Sinus rhythm was restored in all patients.

Where sinus rhythm was restored, electrophysiological study was resumed within 20 minutes of class 1C drug loading, and atrial fibrillation was induced during class 1C drug administration. After five minutes of observation 
Table 1 Age and basic electrophysiological characteristics of patients with atrial fibrillation

\begin{tabular}{|c|c|c|c|c|c|c|c|}
\hline Subjects & $\begin{array}{l}\text { Age } \\
\text { (years) }\end{array}$ & $\begin{array}{l}\text { Basal } \\
\text { cycle } \\
\text { (ms) }\end{array}$ & $\begin{array}{l}\text { Pduration } \\
\text { (ms) }\end{array}$ & $\begin{array}{l}\text { PA duration } \\
\text { (ms) }\end{array}$ & $\begin{array}{l}\text { AH duration } \\
\text { (ms) }\end{array}$ & $\begin{array}{l}\text { HV duration } \\
(\mathrm{ms})\end{array}$ & $\begin{array}{l}\text { cSNRT duration } \\
(m s)\end{array}$ \\
\hline $\mathrm{AF} 1$ & 32 & 1020 & 100 & 60 & 90 & 45 & 400 \\
\hline $\mathrm{AF} 2$ & 32 & 785 & 95 & 40 & 90 & 45 & 250 \\
\hline $\mathrm{AF} 3$ & 40 & 850 & 90 & 50 & 70 & 35 & 240 \\
\hline $\mathrm{AF} 4$ & 44 & 720 & 100 & 60 & 60 & 40 & 330 \\
\hline AF 5 & 33 & 900 & 90 & 45 & 95 & 40 & 260 \\
\hline $\mathrm{AF} 6$ & 37 & 950 & 90 & 60 & 41 & 41 & 280 \\
\hline $\mathrm{AF} 7$ & 41 & 860 & 100 & 26 & 35 & 35 & 340 \\
\hline $\mathrm{AF} 8$ & 32 & 1120 & 85 & 54 & 35 & 35 & 460 \\
\hline $\mathrm{AF} 9$ & 46 & 830 & 75 & 36 & 42 & 42 & 320 \\
\hline AF 10 & 29 & 1080 & 115 & 28 & 36 & 36 & 210 \\
\hline Mean & 36 & 911 & 94 & 46 & 59 & 39 & 309 \\
\hline SD & 5 & 130 & 11 & 13 & 25 & 4 & 77 \\
\hline
\end{tabular}

$\mathrm{AH}$, atrium to bundle of His; cSNRT, corrected sinus mode recovery time; HV, bundle of His to ventricle.

to ensure stability, isoprenaline was resumed and the measurements repeated at steady state. Finally they were repeated after isoprenaline washout on termination of atrial fibrillation.

\section{STATISTICAL ANALYSIS}

The behaviour of $\mathrm{MFF}$ at each recording site and of the corresponding RR interval was investigated by analysis of variance in baseline atrial fibrillation episodes (onset $v 5 \mathrm{~min} v$ isoprenaline $v$ class $1 \mathrm{C}$ drug); when significant differences were found, data were analysed by the Bonferroni test.

The behaviour of MFF and RR interval in atrial fibrillation episodes induced after drug administration was evaluated by the same method (onset $v$ isoprenaline $v$ termination).

Atrial effective refractory periods at baseline and after class $1 \mathrm{C}$ drugs were compared by paired $t$ test.

\section{Results}

Age and basic electrophysiological characteristics are given in table 1 . The latter were within normal limits. The mean (SD) duration of the 10 atrial fibrillation episodes induced at baseline was 36.17 (7.22) minutes (range 28.07 to 48.53 minutes); the duration of the 10 episodes induced after class $1 \mathrm{C}$ drug loading was 22.98 (3.75) minutes (range 18.10 to 29.23 minutes). Each patient had already had self terminating atrial fibrillation episodes induced by the same protocol: two episodes in four patients, three episodes in five patients, four episodes in one patient.

Atrial fibrillation lasting longer than five minutes was induced in all the patients by the single extrastimulus technique under basal conditions. The MFF showed continuous transitions from type I to type II and sometimes type III atrial fibrillation, according to Wells et $a l^{13}$ although a predominance of types I and II was observed in all the patients. All these 10 atrial fibrillation episodes were

Table 2 Effect of intravenous isoprenaline and class $1 C$ drugs on mean of 100 consecutive atrial fibrillation intervals $(M F F)$ and mean RR interval under basal conditions

\begin{tabular}{lllll}
\hline & Baseline & 5 Minutes & Isoprenaline & Class 1C drug \\
\hline MFF HRA (ms) & $177(22)^{\star} \dagger$ & $162(16)^{\star}$ & $144(11)^{\star}$ & $295(49)$ \\
MFF CS (ms) & $180(16)^{\star} \dagger$ & $168(11)^{\star}$ & $148(13)^{\star}$ & $300(47)$ \\
RR (ms) & $480(105)$ & $480(108)$ & $377(37)$ & $529(138) \dagger$ \\
\hline
\end{tabular}

${ }^{\star} \mathrm{p}<0.05 v$ class 1C drug; $+\mathrm{p}<0.05 v$ isoprenaline.

$\mathrm{CS}$, coronary sinus; HRA, high right atrium. terminated by class $1 \mathrm{C}$ drug administration within 10 minutes of intravenous loading (average 6.37 (2.25) minutes, range 2.63 to 8.87 minutes). The MFF shortened after five minutes and showed further shortening during steady state isoprenaline infusion, while it showed a significant increase after class 1C drug administration $(\mathrm{p}<0.02$ by analysis of variance; table 2 , fig 1 ).

The corresponding RR interval was significantly shortened by isoprenaline infusion, whereas no changes were observed between baseline, five minutes, and class 1C drug administration $(p<0.005$ by analysis of variance).
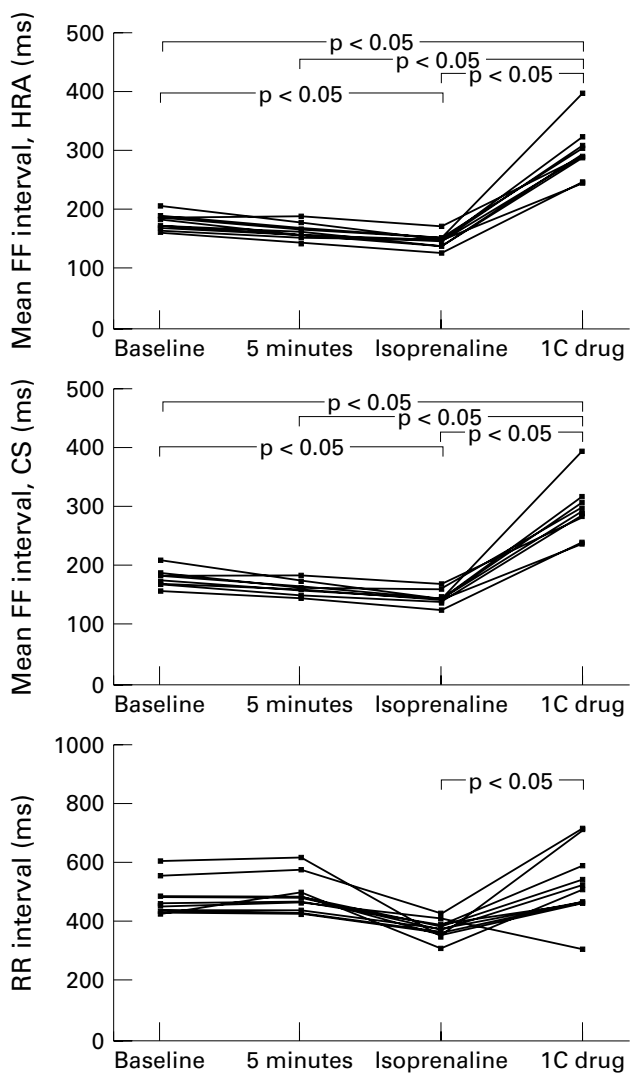

Figure 1 Dynamic behaviour of the MFF and the RR interval through each AF episode from onset, through isoprenaline administration, until termination after class $1 C$ drug administration. From top to bottom: high right atrium (HRA), coronary sinus (CS), and right ventricle in all patients. MFF, mean of 100 consecutive FF intervals; RR, ventricular cycle. 
Table 3 Atrial fibrillation (AF) behaviour and isoprenaline effect during class $1 C$ drug treatment

\begin{tabular}{llll}
\hline & AF onset & Isoprenaline & AF termination \\
\hline MFF HRA (ms) & $269(49)$ & $223(43)$ & $279(46)^{\star}$ \\
MFF CS (ms) & $274(49)$ & $228(41)$ & $282(48)^{\star}$ \\
RR (ms) & $509(92)^{\star}$ & $347(55)$ & $515(79)^{\star}$ \\
\hline
\end{tabular}

${ }^{\star} \mathrm{p}<0.05 v$ isoprenaline

$\mathrm{CS}$, coronary sinus; HRA, high right atrium; MFF, mean FF interval; RR, mean RR interval.

No correlation was found between the dispersion of atrial conduction $\left(\Delta \mathrm{A}_{1} \mathrm{~A}_{2}\right)$, atrial refractoriness, or latent vulnerability index at baseline and the duration of atrial fibrillation or the time to conversion to sinus rhythm after class 1C drug administration. No significant relation was observed between the MFF at induction of atrial fibrillation and time to conversion to sinus rhythm.

Atrial fibrillation was later induced in all 10 patients: in four by programmed stimulation by three extrastimuli at $400 \mathrm{~ms}$ cycle length; in three by two extrastimuli at $600 \mathrm{~ms}, 500 \mathrm{~ms}$, and $500 \mathrm{~ms}$, respectively; and in three by atrial pacing. A value for the atrial effective refractory period was available in six patients at $600 \mathrm{~ms}$ for comparison under basal condition and after class 1C drug administration; it was significantly longer after the class 1C drug (207 (13) $v 192$ (13) ms, p < 0.002). In these six patients, the latent vulnerability index was only minimally changed compared with baseline $(2.18(0.25) \mathrm{cm}$ on drug $v 2.41(0.2) \mathrm{cm}$ at baseline, NS), as was the value for $\Delta \mathrm{A}_{1} \mathrm{~A}_{2}$ (31 (15) ms on drug $v 38$ (13) $\mathrm{ms}$ at baseline, NS).
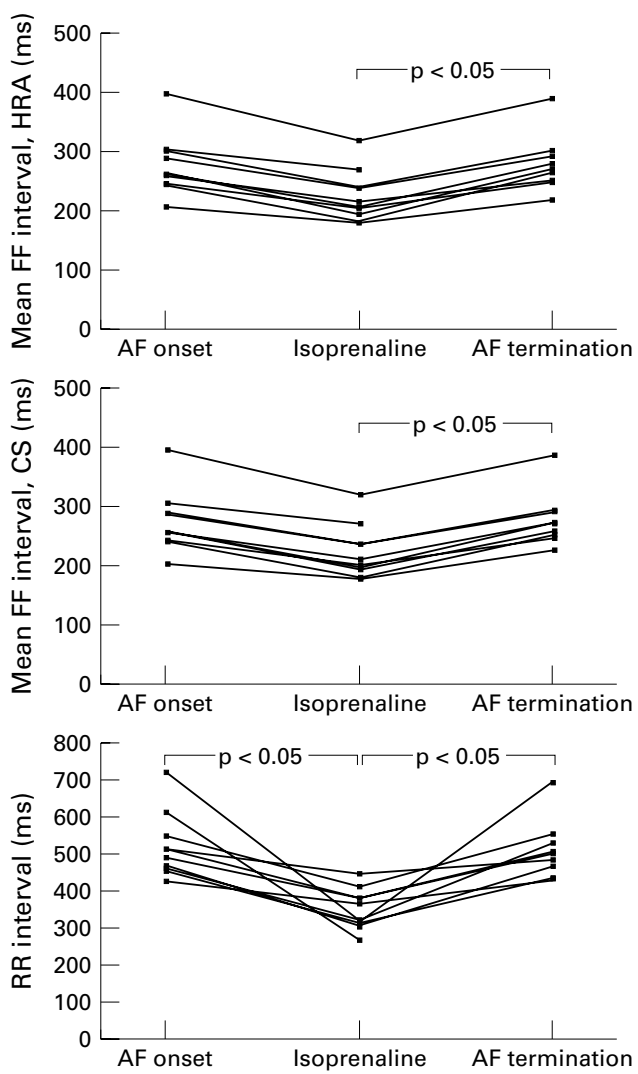

Figure 2 Isoprenaline effect during atrial fibrillation $(A F)$ induced during class $1 C$ drug administration. From top to bottom: high right atrium (HRA), coronary sinus (CS), and right ventricle in all patients. MFF, mean of 100 consecutive FF intervals; $R R$, ventricular cycle.

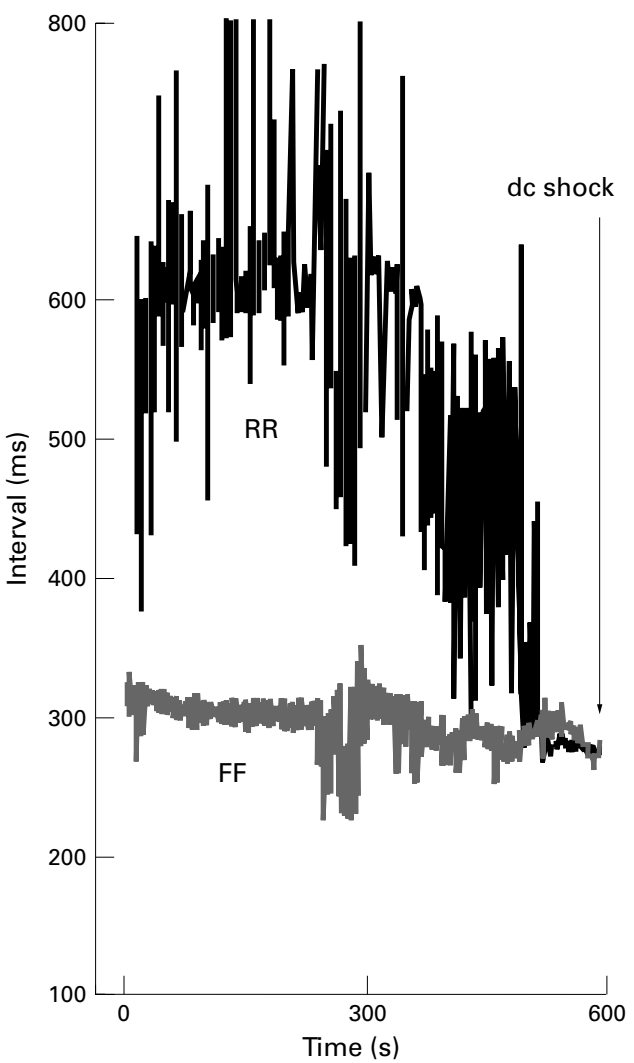

Figure 3 Recordings from the patient who developed haemodynamic collapse while receiving propafenone; isoprenaline administration was started after $300 \mathrm{~s}$ at 5 $\mu \mathrm{g} / \mathrm{min}$, and 1:1 synchronisation at 222 beats/min occurred nearly 300 s later. Time plot of RR (black tracing) and FF intervals (grey tracing) is shown. FF, atrial cycle; RR, ventricular cycle.

The AV node functional refractory period was 329 (21) $\mathrm{ms}$ at baseline and 333 (27) on class $1 \mathrm{C}$ drug at $600 \mathrm{~ms}$ (NS).

The MFF in atrial fibrillation episodes induced after class $1 \mathrm{C}$ drug loading was much longer than was observed at the onset of baseline atrial fibrillation, whereas the RR interval was similar; at steady state isoprenaline administration, both the MFF and the RR interval shortened, whereas they both increased after isoprenaline washout before termination of atrial fibrillation ( $\mathrm{p}<0.03$ for MFF, $\mathrm{p}<0.001$ for RR by analysis of variance; table 3 , fig 2 ).

Isoprenaline administration after $1 \mathrm{C}$ drugs resulted in a trend towards atrioventricular synchronisation, owing to the greater effect on atrioventricular conduction than on the MFF. Sustained 1:1 atrioventricular conduction occurred in two patients, at $320 \mathrm{~ms}$ (187 beats/ $\mathrm{min}$ ) and $270 \mathrm{~ms}$ (222 beats/min), respectively. In the first instance the patient (given flecainide) experienced only mild hypotension and recovered uneventfully shortly after discontinuation of isoprenaline, whereas the second patient (given propafenone) required electrical cardioversion for haemodynamic collapse owing to persistent 1:1 conduction (fig 3 ). The $\mathrm{MFF}$ at atrial fibrillation onset was $397 \mathrm{~ms}$ in the former, whereas it was $304 \mathrm{~ms}$ in the latter. These two patients had shorter Wenckebach cycles by ramp atrial pacing $(310$ and $280 \mathrm{~ms}$, 
respectively) compared with the others (average 338 (17) ms).

\section{Discussion}

In this study we investigated the mechanisms involved in the termination of atrial fibrillation by class $1 \mathrm{C}$ drugs, which appear to be an effective treatment for recent onset atrial fibrillation - as widely reported ${ }^{78^{16-18}}$-in patients with or without structural heart disease. ${ }^{6}$ Although the likelihood of spontaneous conversion to sinus rhythm in patients with recent onset atrial fibrillation seems quite high in the absence of severe heart disease and symptoms of heart failure, ${ }^{6}$ a significant proportion of patients require hospital admission, electrical cardioversion, and anticoagulant treatment in the absence of fast and effective pharmacological treatment. Experimental studies have shown that "atrial fibrillation begets atrial fibrillation," implying that prompt termination of atrial fibrillation is mandatory for long term preservation of sinus rhythm. Thus, understanding the electrophysiological mechanisms associated either with self termination or drug induced termination of atrial fibrillation is of the greatest importance.

In a previous study, ${ }^{9}$ we observed that self terminating atrial fibrillation episodes are associated with prolongation of the MFF, whereas long lasting atrial fibrillation episodes behave the opposite; moreover, the MFF was closely correlated with atrial functional refractoriness.

In this study, the administration of flecainide and propafenone during prolonged episodes of atrial fibrillation-after MFF had already shortened compared with baseline-resulted in a significant prolongation of the MFF followed by arrhythmia termination within 10 minutes in all patients. Thus class $1 \mathrm{C}$ drugs reversed the spontaneous trend in the MFF, leading to an increase in MFF which was associated with termination of atrial fibrillation. Interestingly, the prolongation of $\mathrm{MFF}$ occurred to a much greater extent (average $300 \mathrm{~ms}$ ) than previously observed $^{9}$ in self terminating episodes (average $198 \mathrm{~ms}$ ).

The interpretation of the drug effect is inherently difficult as these agents markedly decrease conduction velocity as well as increasing atrial refractoriness at high rates in a use dependent manner, ${ }^{19} 20$ and both mechanisms prolong the MFF. Nonetheless, the MFF itself is the most useful index in the clinical electrophysiology setting because first, the effect of class $1 \mathrm{C}$ drugs on atrial refractoriness is much greater during atrial fibrillation than in sinus rhythm owing to use dependency; second, there is no practical way of evaluating conduction velocity in this setting. Examining the dynamic behaviour of the MFF, one could argue that class 1C drugs reverse the progressive shortening of the MFF associated with persistence of atrial fibrillation and thus enhance the likelihood of conversion to sinus rhythm. The electropharmacological basis could either be a conduction block of depolarising wavefronts in a critical area, or the use dependent prolongation of atrial refractoriness to a critical wavelength value at which arrhythmia perpetuation is impossible. The greater efficacy of class 1C drugs compared with quinidine, ${ }^{21}$ which lacks this latter characteristic, ${ }^{20}$ supports the second hypothesis.

In a recently published paper, ${ }^{22}$ Stambler et al reported the electrophysiological effects of procainamide and ibutilide in the conversion to sinus rhythm of 48 patients with long standing atrial fibrillation (average duration 23 (20) days), 46 of whom had type I atrial fibrillation according to Wells et al. ${ }^{13}$ Comparison of the effect on atrial fibrillation cycle and on the monophasic action potential duration (MAPD) showed that the two drugs were equally effective in prolonging the atrial fibrillation cycle, but ibutilide increased MAPD to a greater extent. This was associated with a significantly greater efficacy in restoring sinus rhythm (9/28) than with procainamide $(1 / 20)$. These data support the hypothesis that prolongation of refractoriness may be the most important mechanism whereby conversion to sinus rhythm is achieved during antiarrhythmic treatment, at least in type I chronic atrial fibrillation. One important characteristic in the population studied by Stambler et al was the predominance of type I atrial fibrillation (which has a regular atrial pattern with isoelectric intervals between consecutive depolarisations), ${ }^{22}$ whereas in patients with chronic atrial fibrillation, type II and III variants are more likely to be observed owing to electrophysiological remodelling which leads to shortening of atrial refractoriness. ${ }^{5}$

In our patients, dynamic changes from type I to type II and III were continuously recorded at the same site at different times, although the duration of atrial fibrillation did not exceed 50 minutes. In addition, the MFF after five minutes in our patients was exactly similar to that observed by Stambler et al in their population with chronic atrial fibrillation, ${ }^{22}$ thus implying a greater derangement of atrial electrophysiological properties in our patients. This underlines the fact that the pathological substrate and the electrophysiological milieu are very different in lone atrial fibrillation and in structural heart disease, with obvious implications for the efficacy of antiarrhythmic treatment.

The efficacy of class $1 \mathrm{C}$ drugs in recent onset atrial fibrillation may thus be explained either by a frequency dependent increase in atrial refractoriness or by conduction block in an "electrophysiologically ill" tissue. The lesser efficacy of ibutilide in chronic atrial fibrillation observed by Stambler et al results from the arrhythmia duration, which causes further electrophysiological remodelling by shortening atrial refractoriness and MFF; this implies that prolongation of refractoriness is mandatory for an antiarrhythmic action. As pointed out by Stambler et al, ${ }^{22}$ a critical value of MFF (below $160 \mathrm{~ms}$ ) or MAPD (below $125 \mathrm{~ms}$ ) seems to rule out the possibility of restoring sinus rhythm by pharmacological treatment. This observation reinforces the concept that atrial fibrillation of recent onset should be terminated promptly, with a view to preventing the electrophysiological remodelling that occurs 
with time ${ }^{5}$ and which in turn makes it less likely that sinus rhythm can be restored.

ISOPRENALINE EFFECT DURING ATRIAL

FIBRILLATION

To our knowledge, no data have been reported on the effect of isoprenaline on the atrial cycle during atrial fibrillation. Our data clearly show that isoprenaline shortens the MFF, either in the drug-free state or after class $1 \mathrm{C}$ drugs. This is of great importance when considering either the setting of acute loading for the treatment of atrial fibrillation, or recurrence of atrial fibrillation during chronic treatment-if adrenergic stimulation occurs, the combination of long $\mathrm{MFF}$ and enhanced AV conduction may result in 1:1 synchronisation with fast ventricular rates and, below a certain MFF value, haemodynamic collapse. This phenomenon has been emphasised before, ${ }^{10} 112324$ but its electrophysiological basis was not described; is likely to occur during atrial fibrillation in subjects with normal left ventricular function, who usually have intact $\mathrm{AV}$ conduction properties and short Wenckebach cycles. Thus an isoprenaline challenge is a useful test to identify the electrophysiological mechanism of unexpected outcomes such as syncope or wide QRS complex tachycardia during treatment with a class $1 \mathrm{C}$ drug. Administration of agents that can modulate AV conduction (calcium antagonists or $\beta$ blockers plus digoxin) has been proposed for reducing the risk of atrial flutter with 1:1 AV conduction after class $1 \mathrm{C}$ agents, ${ }^{11}$ but this concept has never been supported by a prospective trial. In fact, the occurrence of such a phenomenon depends on several factors, such as the autonomic input to the atria and the AV node, and the pharmacological action of class $1 \mathrm{C}$ drugs on both the MFF and the AV node (which mainly depends on age and underlying heart disease). It is conceivable that the presence of a short Wenckebach cycle, a structurally normal heart, and prolongation of the MFF in the range of 270-300 ms may predispose selected patients to very rapid ventricular rates (200-220 beats/min) in cases of $1: 1$ synchronisation.

A shift of the electrocardiographic pattern from atrial fibrillation to atrial flutter has been described even in patients not treated with antiarrhythmic agents ${ }^{625}$; thus class $1 \mathrm{C}$ drugs may simply emphasise or unmask a peculiar electrophysiological pattern which may be patient or arrhythmia related. An issue for future study could be whether the most appropriate antiarrhythmic treatment for atrial fibrillation-either in terms of efficacy or safety-can be selected on the basis of the electrophysiological pattern, which may range from a regular flutter-like activity (type I) to a rather disorganised (type II and III) atrial activation with frequent transitions from one to the other.

LIMITATIONS OF THE STUDY

The population under evaluation represents a minority of the wide clinical spectrum of patients with atrial fibrillation, but in this selected group with lone paroxysmal atrial fibrillation the evaluation of atrial electrophysiological derangement is enhanced because of the absence of gross anatomical alterations. Investigation of the dynamic behaviour of atrial fibrillation is crucial to our understanding of the mechanisms that underlie the transition from the paroxysmal to the chronic form, and may provide guidance for new pharmacological and non-pharmacological treatments, for example radiofrequency ablation. A more extensive and sophisticated mapping approach than recording the HRA and CS electrogramssuch as that provided by multisite recording catheters or by surgical mapping of the atriawould have certainly been helpful; however, any added benefit in terms of monitoring the effects of pharmacological treatment is as yet undefined.

1 Allessie MA, Lammers WJEP, Bonke FIM, et al. Experimental evaluation of Moe's multiple wavelet hypothesis of atrial fibrillation. In: Zipes DP, Jalife J, eds. Cardiac electrophysiology and

2 Rensma PL, Allessie MA, Lammers WJEP, et al. Length of excitation wave and susceptibility to reentrant atrial rrhythmias in normal conscious dogs. Circ Res 1988;62: 395-410.

3 Allessie M, Kirchhof C, Scheffer GJ, et al. Regional control of atrial fibrillation by rapid pacing in conscious dogs. Circulation 1991;84:1689-97.

4 Kirchhof C, Chorro F, Scheffer GJ, et al. Regional entrainment of atrial fibrillation studied by high-resolution mapping in open chest dogs. Circulation 1993;88:736-49.

5 Wiiffels MCEF, Kirchhof CJHJ, Dorland R, et al. Atrial fibrillation begets atrial fibrillation. A study in awake chronically instrumented goats. Circulation 1995;92:195468.

6 Boriani G, Biffi M, Capucci A, et al. Oral propafenone to convert recent-onset atrial fibrillation in patients with and without underlying heart disease. Ann Intern Med 1997; 126:621-5.

7 Donovan KD, Power BM, Hockings BEF, et al. Intravenous flecainide versus amiodarone for recent-onset atrial fibrillation. Am f Cardiol 1995;75:693-7.

8 Capucci A, Boriani G, Botto GL, et al. Conversion of recent-onset atrial fibrillation by a single oral loading dose of propafenone or flecainide. Am f Cardiol 1994;74:503-5.

9 Capucci A, Biffi M, Boriani G, et al. Dynamic electrophysiologic behaviour of human atria during paroxysmal atrial fibrillation. Circulation 1995; 92:1193-202.

10 Naccarelli GV, Rinkenberger RL, Dougherty AH, et al. Occurrence of atrial flutter with $1: 1$ AV nodal conduction
during encainide and flecainide therapy [abstract]. Circulation 1989;80(suppl II):II-634.

11 Marcus FI. The hazards of using type 1C antiarrhythmic drugs for the treatment of paroxysmal atrial fibrillation. $A m$ f Cardiol 1990;66:366-7.

12 Nollo G, Del Greco M, Ravelli F, et al. ECG-oesophageal recording for beat-to-beat analysis of $\mathrm{A}-\mathrm{V}$ conduction time variability. Computers in cardiology. New York: IEEEComputer Society Press, 1992:433-6.

13 Wells JL, Kouchoukos NT, MacLean WAH, et al. Characterisation of atrial fibrillation in man: studies following open heart surgery. PACE 1978;1:426-38.

14 Lammers WJEP, Ravelli F, Disertori M, et al. Variations in human flutter cycle length induced by ventricular beats: evidence of a reentrant circuit with a partially excitable gap. f Cardiovasc Electrophysiol 1991;2:375-87.

15 Ravelli F, Disertori M, Cozzi F, et al. Ventricular beats induce variations in cycle length of rapid (type II) atrial flutter in man. Evidence of leading circle reentry. Circulation 1994;89:2107-16.

16 Suttorp MJ, Kingma HJ, Jessurin ER, et al. The value of class $1 \mathrm{C}$ antiarrhythmic drugs for acute conversion of par-
oxysmal atrial fibrillation or flutter to sinus rhythm. $f \mathrm{Am}$ oxysmal atrial fibrillation or
Coll Cardiol 1990;16:1722-7.

17 Boriani G, Capucci A, Lenzi T, et al. Propafenone for conversion of recent-onset atrial fibrillation. A controlled comparison between oral loading dose and intravenous administration. Chest 1995;108:355-8.

18 Capucci A, Lenzi T, Boriani G, et al. Effectiveness of loading oral flecainide for converting recent-onset atrial fibrillation to sinus rhythm in patients without structural heart disease or with only systemic hypertension. Am f Cardiol 1992;70:69-72.

19 Wang J, Bourne GW, Wang Z, et al. Comparative mechanisms of antiarrhythmic drug action in experimental mechan fibrillation. Circulation 1993;88:1030-44.

20 Wang Z, Pelletier LC, Talajic M, et al. Effects of flecainide and quinidine on human atrial action potentials. Role of and quinidine on human atrial action potentials. Role of
rate-dependence and comparison with guinea pig, rabbit and dog tissues. Circulation 1990;82:274-83. 
21 Capucci A, Boriani G, Rubino I, et al. A controlled study on oral propafenone versus digoxin plus quinidine in converting recent onset atrial fibrillation to sinus rhythm. Int $\mathcal{F}$ Cardiol 1994;43:305-13.

22 Stambler BS, Wood M, Ellenbogen KA. Antiarrhythmic actions of intravenous ibutilide compared with procainamide during human atrial flutter and fibrillation. Circulation 1997;96:4298-306.
23 Murdock CJ, Kyles AE, Yeung-Lai Wah JE, et al. Atrial flutMurdock CJ, Kyles AE, Yeung-Lai Wah JE, et al. Atrial flut-
ter in patients treated for atrial fibrillation with propafter in patients treated for atrial fib

24 Feld GK, Chen PS, Nicod P, et al. Possible atrial proarrhythmic effects of class $1 \mathrm{C}$ antiarrhythmic drugs. $\mathrm{Am}$ f Cardiol 1990;66:378-83.

25 Botto GL, Bonini W, Broffoni T, et al. Regular ventricular rhythms before conversion of recent onset atrial fibrillation to sinus rhythm. Pace 1994;17:2114-17.

\section{Management of superior vena caval obstruction secondary to a pacing wire with percutaneous intravascular stent insertion}

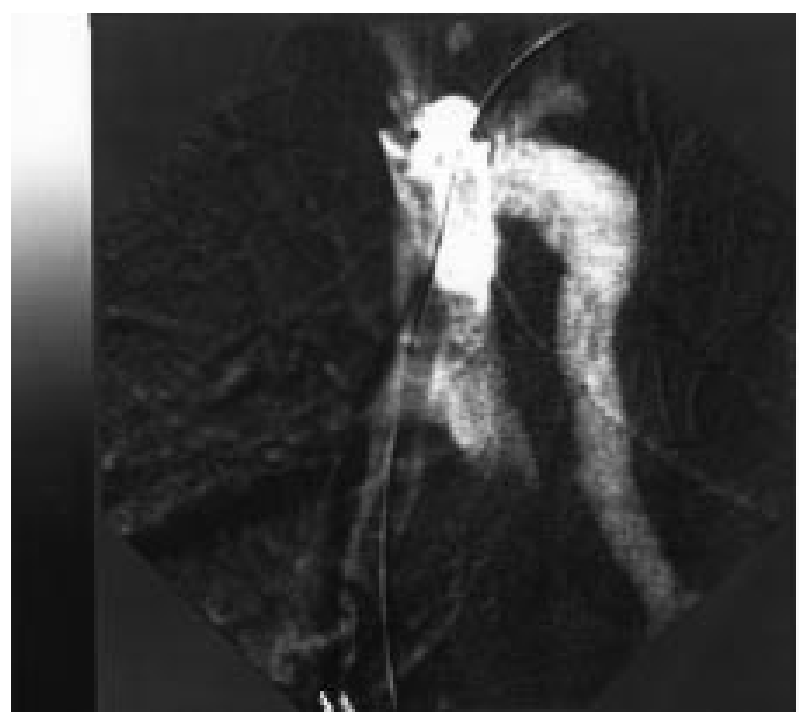

Superior vena caval (SVC) syndrome following permanent pacemaker implantation is an uncommon complication and may occur as a consequence of thrombosis, stenosis or both. Accepted modes of treatment include thrombolysis, surgery, and percutaneous transluminal coronary angioplasty (PTCA).

A 77 year old man presented 8 years after AAI pacemaker insertion with a six month history of progressive dyspnoea and facial oedema. On examination he had the classic findings of SVC obstruction. Contrast enhanced computed tomography confirmed an intravascular SVC filling defect enveloping the pacing wire extending from the left brachiocephalic vein to right atrium.

Tissue plasminogen activator administration $(90 \mathrm{mg}$ over 2 hours) followed by 48 hour heparin infusion failed to recanalise the vessel. Right heart catheterisation confirmed complete SVC occlusion with drainage via the azygous system

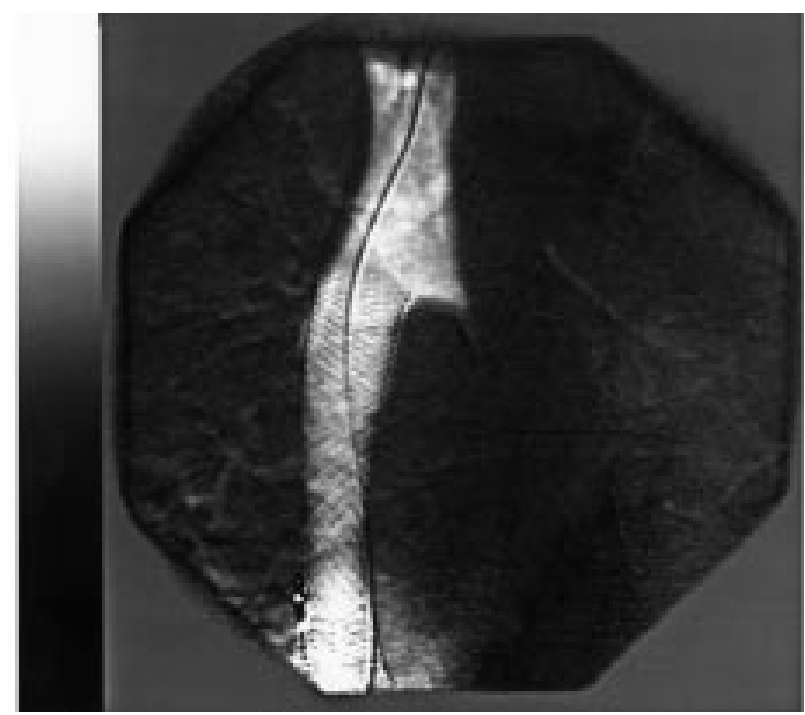

(left). A $12 \mathrm{~mm}$ angioplasty balloon was successfully inflated at the right atrium/SVC junction but deflation resulted in immediate re-occlusion. A $16 \times 56 \mathrm{~mm}$ self expanding Wallstent was inserted and dilated with a $20 \mathrm{~mm}$ valvotomy balloon. Subsequent angiography demonstrated free flow of contrast into the right atrium (right). Oral ticlopidine (250 mg bid) was given for two weeks and the patient was prescribed warfarin. Pacing checks before and after the procedure were unremarkable. At three months he remained asymptomatic.

Fear of pacing wire damage has resulted in underuse of stents in treating SVC syndrome with only one previous report of such a procedure. This report demonstrates that stents can be used safely in resistant cases.

N MELIKIAN C I O BROOKES A F RICKARDS 